\title{
Operant Conditioning of Head-Waving in Aplysia. III. Cellular Analysis of Possible Reinforcement Pathways
}

\author{
David G. Cook and Thomas J. Carew \\ Departments of Psychology and Biology, Yale University, New Haven, Connecticut 06520
}

\begin{abstract}
Operant conditioning of the head-waving response in Aplysia, as well as conditioning of the electrical activity of identified neck muscles, can be induced readily when bright light is used as aversive reinforcement (Cook and Carew, 1986, 1989b). A cellular analysis of this type of operant conditioning requires an understanding of the neural circuitry that underlies the reinforcement pathways involved in the conditioning. In the present paper we describe a cellular analysis of possible reinforcement pathways that mediate the aversive effects of bright light in the CNS of Aplysia.

Using a semi-intact "split-body" preparation, we explored the effects of bright light on the operant response pathway by recording intracellularly from identified pedal neck and body wall motor neurons, which contribute to the operant response. In these experiments we identified 2 light-sensitive pathways. One pathway, from the eyes and rhinophores, mediated functional inhibition of light-induced excitation of pedal motor neurons. The other pathway, from the oral veil (cerebral ganglion nerves $\mathrm{C}_{1-\mathrm{C}}$ ) mediated significant excitation of the same motor cells. Randomly occurring blank trials ruled out the possibility that the light-induced effects were due to sampling bias. Finally, surgical isolation of the CNS from the periphery showed that none of the effects of light were due to direct illumination of central neurons. This identification of candidate reinforcement pathways will facilitate a cellular analysis of operant conditioning of headwaving in Aplysia.
\end{abstract}

Aplysia californica can rapidly learn to modify their head-waving behavior operantly in response to contingent reinforcement. Animals learn to reduce the amount of time spent head-waving on one side of their body to avoid the presentation of bright, diffuse whole-field illumination, a stimulus the animals find aversive (Cook and Carew, 1986). A cellular analysis of this form of operant conditioning requires specifying both the motor output pathway involved in the operant response (the components of the CNS and periphery that mediate head movements) and the reinforcement pathway that produces the operant conditioning (visual input to the CNS). The previous 2 papers focused on an analysis of the operant response pathway. In those

\footnotetext{
Received Dec. 8, 1988; revised Mar. 3, 1989; accepted Mar. 6, 1989.

We wish to thank Frederick Kuenzi and Mark Stopfer for their helpful criticism of the manuscript.

This work was supported by NIH Grant 5-RO1-MH41083 and by NIH BRSG Grant 507-RR-075015 to T.J.C.

Correspondence should be addressed to Thomas J. Carew, Yale University, Departments of Psychology and Biology, Yale Station, 11A, New Haven, CT 06520 .

Copyright $\odot 1989$ Society for Neuroscience $0270-6474 / 89 / 093115-08 \$ 02.00 / 0$
}

studies, we identified a discrete set of bilaterally symmetric muscles in the neck and body wall, the lateral columellar muscles (LCMs), whose electromyographic activity is significantly correlated with the component of head-waving (the horizontal component) that is modified by operant conditioning (Cook and Carew, 1989a) and is modified by contingent presentation of the same reinforcement (bright light) that produces operant conditioning of the head-waving response (Cook and Carew, 1989b).

In the present paper, we describe an analysis of candidate reinforcement pathways from the periphery to the CNS. We were specifically interested in identifying and characterizing visual pathways that modulate the activity of the motor output pathway for head-waving. Since the operant response involves activation of neck and body wall muscles (including the LCMs), identified motor neurons in the pedal ganglion that innervate the neck and body wall (Hening et al., 1979) provided a useful cellular vantage point from which to assess the effects of reinforcement (bright light) on the operant response pathway. Therefore, we recorded intracellularly from neck and body wall motor neurons and delivered the same type of whole-field illumination that produces operant conditioning of both the head-waving response (Cook and Carew, 1986) and the EMG activity of the LCMs (Cook and Carew, 1989b). Using this approach we were able to identify 2 light-sensitive pathways with functionally opposite effects: One pathway, from the eyes and rhinophores (the optic and rhinophore nerves), mediated significant inhibition of light-induced excitation in the pedal motor neurons; the other pathway, from the head and oral veil region [the superior labial, anterior tentacular, and inferior labial nerves (nerves C1-C3)], mediated significant excitation in the same motor neurons. The identification and characterization of these candidate reinforcement pathways now provides the opportunity to examine sites of interaction between operant response and visual pathways within the CNS of Aplysia.

Some of the results described in this paper have been reported in abstract form (Cook and Carew, 1987).

\section{Materials and Methods}

Animals. Sixteen adult $A$. californica (150-250 gm) from Sealife Supply (Sand City, CA) were used. Animals were individually housed in a 1140 liter aquarium filled with circulating, aerated artificial seawater (Instant Ocean: Aquarium Systems, Mentor, $\mathrm{OH}$ ) maintained at room temperature $\left(20-22^{\circ} \mathrm{C}\right)$.

Split-body preparation. Animals were prepared for intracellular recordings from pedal neurons using a modified split-foot preparation (Hening et al., 1979; Carew et al., 1981). Animals were anesthetized by injection of isotonic $\mathrm{MgCl}_{2}$ into the body cavity ( $50 \%$ of their body weight). The anterior ventral and dorsal body wall was cut longitudinally to expose the CNS. The buccal mass and buccal ganglion were then removed. In the process of removing the buccal mass, the cephalic artery was first ligated and then cut. The anterior aorta was then cut imme- 


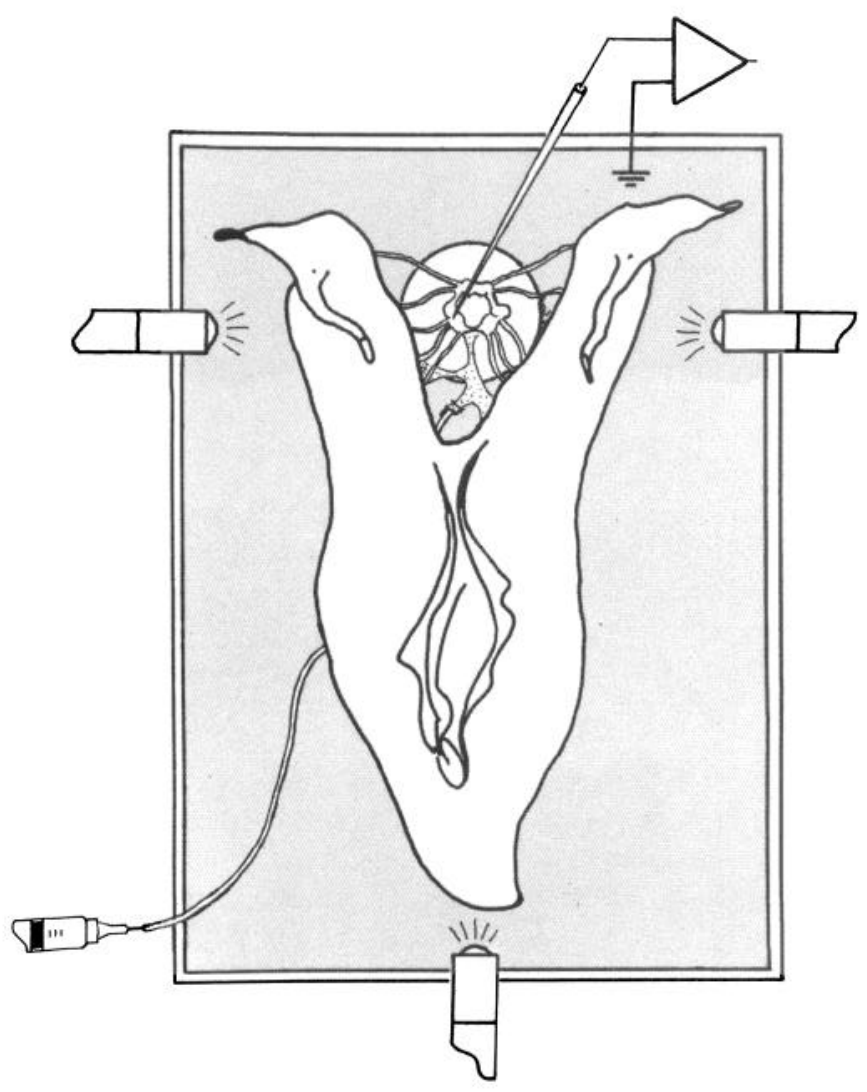

Figure 1. Experimental preparation. A "split-body" preparation was used to record intracellularly from central neurons (with the CNS intact and connected to the periphery) to assess the effects of whole-body illumination delivered by fiberoptic light guides.

diately rostral to its junction with the abdominal aorta. A length of polyethylene tubing (PE 90 Intramedic, Clay Adam, Parsippany, NJ) was installed in the rostral end of the anterior aorta. Thus, the circulatory system of the animal could be perfused with Aplysia Ringer's solution $\left(460 \mathrm{~mm} \mathrm{NaCl}, 55 \mathrm{~mm} \mathrm{MgCl}_{2}, 11 \mathrm{~mm} \mathrm{CaCl}_{2}, 10.4 \mathrm{~mm} \mathrm{KCl}, 10 \mathrm{~mm}\right.$ Trizma) to flush out the $\mathrm{MgCl}_{2}$ that accumulated in the interstitial space in earlier phases of the dissection. The perfusion procedure was carried out for at least 10 min prior to beginning the experiment. Next, the body wall was pinned back to hold it in place and to expose the CNS. To prevent body wall movements from dislodging intracellular electrodes, each ganglion in the circumeseophogeal ring, as well as all peripheral nerves, was pinned securely through the sheath. The 2 pedal ganglia were then desheathed to expose the clusters of neurons near the statocysts in the dorsal-anterior portion of each ganglion.

Electrophysiological techniques. Using the split-body preparation it was possible to obtain stable intracellular recordings from pedal neurons for many hours while the body wall of the animal exhibited clear movements. Intracellular recordings were accomplished using standard DC amplifiers (Getting model 5A; Iowa City, IA) and glass micropipettes filled with $2 \mathrm{~m}$ potassium citrate. Microelectrodes were bevelled to yield 10-15 M $\Omega$ electrodes. Pedal neurons were classified as candidate motor neurons if (1) when depolarized with injected current they produced a clear movement in the body wall and (2) if electrical stimulation of the appropriate peripheral nerves (via hook electrodes) produced an antidromic action potential in the neuron. Recordings were restricted to large $70-100 \mu \mathrm{m}$ pale neurons clustered near the statocysts of the pedal ganglion (the IIa cluster) (Hening et al., 1979).

Light stimulus protocol. Constant dim background illumination $(0.5$ footcandles) was provided by a red incandescent light source. Photic stimuli in the split-foot preparation was provided by 2 quartz halogen light sources (Dolan Jenner; Woburn, MA) delivered to the preparation via 3 fiberoptic light guides that illuminated the entire preparation. The intensity of the photic stimuli varied across the length of the preparation from 500-700 footcandles. The light source was first passed through a heat filter positioned between the light source and fiberoptic light guides, which minimized light-induced heating of the preparation. During the experiment the preparation was constantly perfused with artificial seawater.

In each experiment the preparation was exposed to 3 light trials ( 30 sec in duration) and 3 blank trials, in which no light was delivered. The light and blank trials were randomly intercalated so that, at the beginning of any trial, the experimenter did not know whether the ensuing trial would be a light or blank trial. Following any light trial, there was a 10 min interval before the next trial, to permit readaptation to dim baseline light conditions. To determine the effect of light on the firing rate of a motor cell the number of action potentials fired by the the cell during the $30 \mathrm{sec}$ light trial was compared with that of the $30 \mathrm{sec}$ immediately preceding the light trial. Following the random delivery of 3 light and 3 blank trials the appropriate nerves were bilaterally transected and the
Figure 2. Schematic diagram of the circumesopheogeal ganglia. Dorsal view of the circumesopheogeal ring ganglia is illustrated. The right pedal ganglion is shown desheathed to illustrate the pedal motor neurons that we record from (shaded black). These motor neurons innervate the neck and body wall. Also illustrated are 2 peripheral pathways that carry photic information into the CNS: (1) the optic and rhinophore nerves; and (2) the oral veil nerves [superior labial $(\mathrm{C} 1)$, anterior tentacular (C2), and inferior labial (C3)].

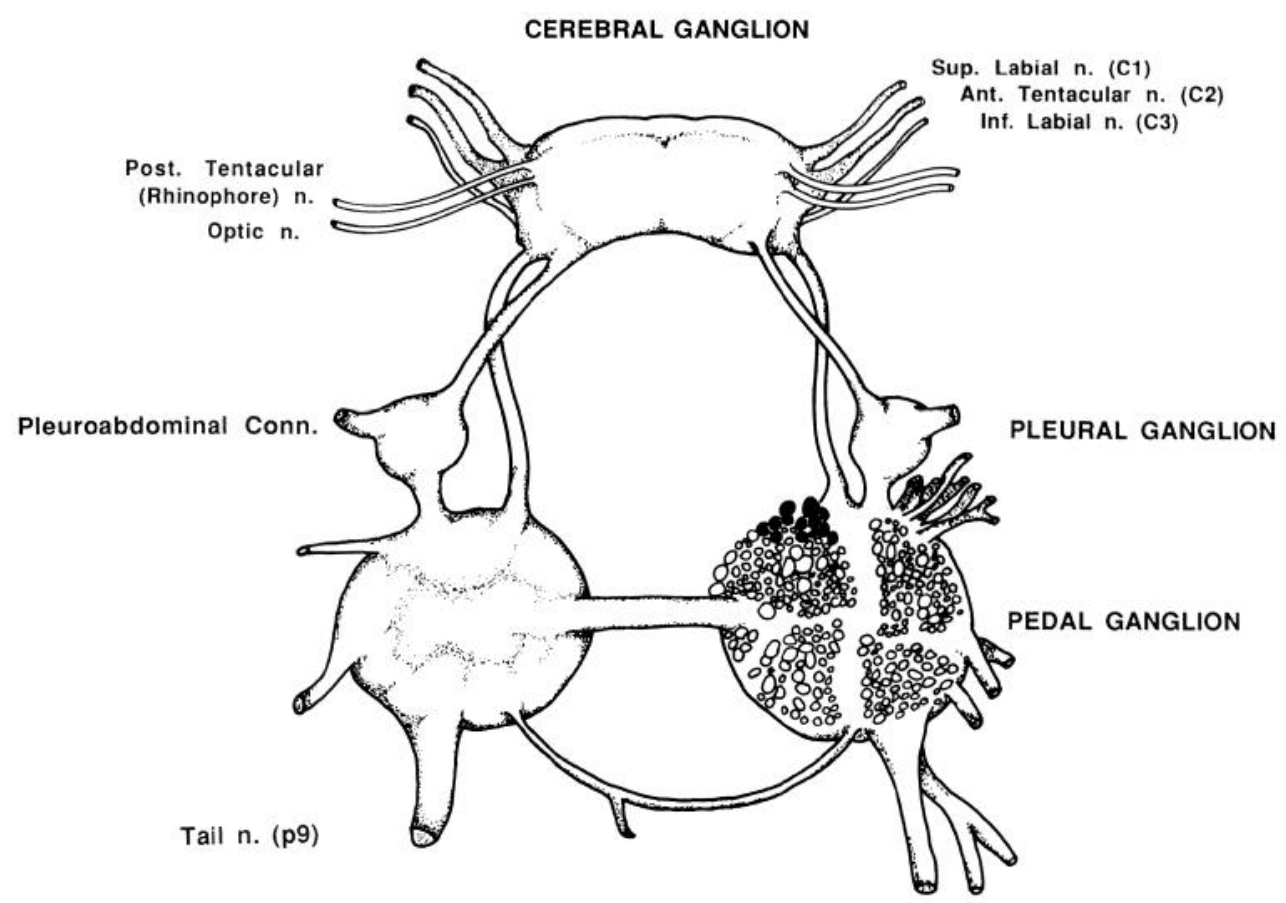


entire procedure was repeated, after a minimum of a 10 min waiting period.

Statistics procedures. Standard $t$-tests were used to analyze the data. All data are expressed as means \pm SEM.

\section{Results}

Bright whole-body illumination can serve as a potent aversive reinforcer in operant conditioning of head-waving in Aplysia (Cook and Carew, 1986, 1989b). The primary goal of the present study was to identify the neural pathways that mediate this reinforcement in the CNS. The preparation we used to investigate this question is illustrated in Figure 1. This "split-body" preparation provides the opportunity to record intracellularly from central neurons, while the intact CNS is connected to the periphery via the peripheral nerves (see Materials and Methods). Thus, using this preparation, the same reinforcement used in our operant conditioning studies (whole-field illumination of the entire body with bright light) can be delivered to the animal, while the effects of this stimulus can be directly assessed in central neurons.

The neurons we chose to examine were a cluster of large motor cells located near the statocysts on the dorsal surface of the pedal ganglion (Fig. 2). These pedal motor cells were selected because they produce powerful contractions of the neck and body wall and are active during a variety of neck movements (Hening et al., 1979); thus, they are likely candidates to contribute to the operant response of head-waving. The purpose of these experiments was 2-fold: First, we wished to determine whether photic stimulation could alter the excitability of these motor cells. Second, if photic stimulation could modulate the motor neurons' activity we wished to detcrmine which peripheral nerves carry visual stimulation to the CNS.

We examined the effects of 2 types of peripheral nerve transections upon light-induced modulation of motor neuron excitability: (1) bilateral transections of the optic and rhinophore nerves and (2) bilateral transections of the oral veil nerves [superior labial (C1), anterior tentacular (C2), and inferior labial (C3) nerves; see Fig. 2]. The protocol we employed to examine the role of these 2 pathways is schematically illustrated in Figure 3. Each cell selected for study (see Materials and Methods for selection criterion) received 2 types of trials. First, with the appropriate nerves to the periphery intact, the preparation received: (1) 3 light trials, each consisting of a $30 \mathrm{sec}$ exposure to bright light, and (2) 3 blank trials, in which no light stimulus was administered. The presentation of light trials and blank trials was randomly mixed. For each trial (light or blank), the number of action potentials during the test trial period was compared with the number of action potentials observed in a baseline phase ( $30 \mathrm{sec}$ immediately preceding each trial). The blank trials served to insure that any systematic changes in a cell's activity during a light trial could be attributed to the presentation of the light stimulus, and not to some other uncontrolled feature of the experiment. Following the 3 light and 3 blank trials, the appropriate peripheral nerves were severed and the identical procedure of randomly delivered light and blank trials was repeated.

\section{Role of optic and rhinophore nerves in light-induced excitation of pedal motor neurons}

In these experiments, the response of the motor neurons to light was first determined with all peripheral nerves to the CNS intact (see Materials and Methods). An example of the effect of light

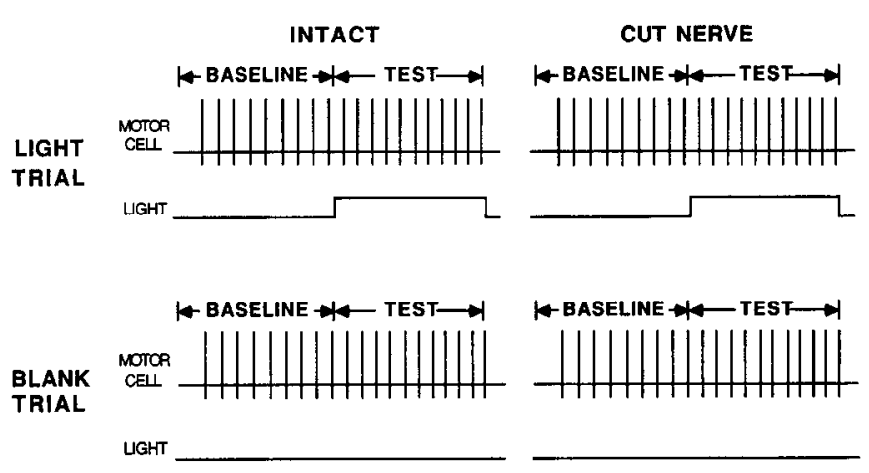

Figure 3. Experimental paradigm. Each trace represents a sample intracellular recording from a pedal motor neuron. Each neuron receives 2 types of trials: light trials and blank trials ( 3 trials each, randomly delivered). Each trial consists of a $30 \mathrm{sec}$ baseline phase and a $30 \mathrm{sec}$ test phase. Both sets of trials are delivered first with all peripheral nerves intact and then again following transection of appropriate peripheral nerves.

on a pedal motor neuron under these conditions is shown in the top trace of Figure 4. In response to light, the motor cell showed a delayed and modest increase in the number of action potentials during the light trial compared with the baseline (an increase of $18 \%$, from 78 to 92 action potentials). The middle trace shows the effect of bilateral transection of the optic and rhinophore nerves on the same cell. The results from this phase of the experiment were surprising (see Discussion). Rather than abolishing the modest light-induced increase in action potential number, as we had expected, following transection of the optic and rhinophore nerves, identical light stimulation produced a striking increase in the number of action potentials (an increase of $43 \%$, from 80 to 115 action potentials). It was possible, since the CNS itself was also exposed to light, that some component of the increased activity during a light trial could be due to the direct effect of light on central neurons (Arvanitaki and Chalazonitis, 1961; Newby, 1972; Block and Smith, 1973). The final phase of the experiment, shown in the bottom trace of Figure 4 , ruled out this possibility, since surgical isolation of the CNS from the periphery completely abolished any effect of light on the motor neuron's firing rate. These results suggest 2 conclusions: First, with the CNS and peripheral nerves intact, the effect of bright light upon the pedal motor cells is modest. Second, following transection of the optic and rhinophore nerves, the pedal motor cells are indeed modulated by photic input. Specifically, sectioning these nerves unmasked a potent light-induced excitation. This observation raised the possibility that the input from the eyes and rhinophores, each a major source of photic input to the CNS (Chase, 1979; Jacklet, 1980), is functionally inhibitory, at least under the conditions of our experiments.

The results of 10 experiments (one motor neuron per preparation) are summarized in Figure 5, which shows the mean change in spike number before and after transection of the optic and rhinophore nerves for both light and blank trials. With the CNS intact, light caused a small increase in the number of action potentials elicited in the cells $(\bar{x}$ increase $=5$ spikes, \pm 3.23 ). Although 8 out of 10 cells showed light-induced increase in firing with the peripheral nerves intact, a within-condition analysis showed that the increase was not statistically significant $(t[9]=$ 1.55 , n.s.). However, following transection of the optic and rhinophore nerves, a within-condition analysis showed that the 


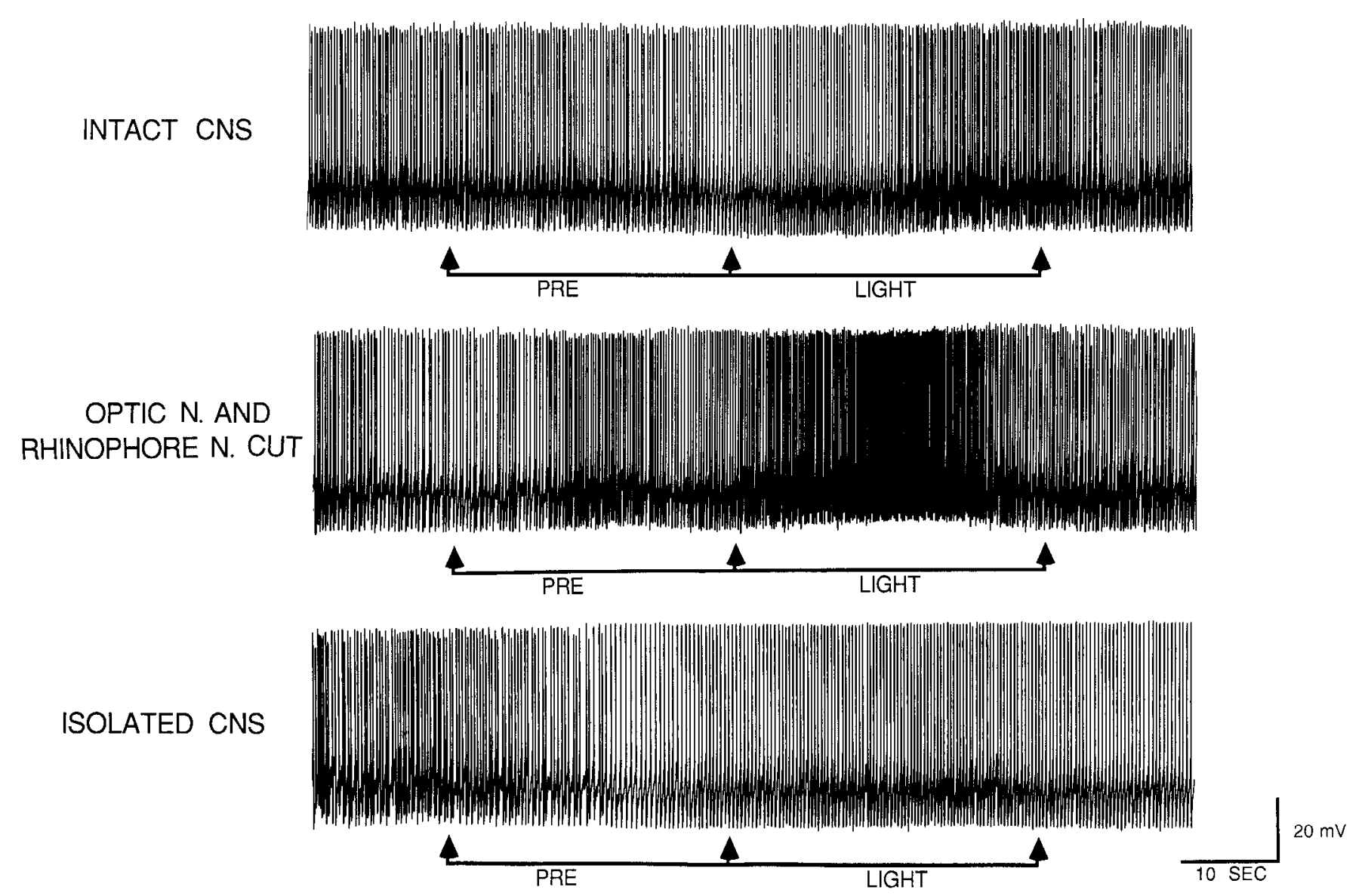

Figure 4. Role of the optic and rhinophore nerves in light-induced excitation of pedal motor neurons. Traces are intracellular recordings from a pedal motor ncuron using the preparation shown in Figure 1. Top trace, With all peripheral nerves intact, light produces a delaycd and modcst increase in motor neuron firing. Middle trace, Following bilateral transection of the optic and rhinophore nerves, light produces a much greater increase in motor neuron firing. Bottom trace, Following complete isolation of the CNS from the periphery, light has no effect on pedal motor neuron firing rate.

motor cells exhibited a significant increase in light-induced firing $(\bar{x}$ increase $=22.5$ spikes, $\pm 6.4 ; t[9]=3.52, p<0.005)$. Moreover, a between-condition analysis showed that the motor cells exhibited a significant increase in their response to light following nerve transection compared with their own response prior to nerve transections $(t[9]=2.79, p<0.01)$. The effects of blank trials both before and after nerve transection were not statistically significant, showing that sampling bias cannot explain these results.

Changes in baseline excitability following nerve transection could, in principle, contribute to the changes in light-induced excitation of the motor cells that we observe. To assess this possibility we examined the baseline firing rate of each motor cell, by comparing the baseline number of action potentials during light and blank trials before and after nerve transection. The mean number of action potentials prior to nerve transection was $60.5( \pm 4.9)$, and following nerve transections the mean number of action potentials was $57.8(+6.57)$. This slight decrease in baseline firing following transection was not significant $(t[9]=0.56$, n.s.). Furthermore, if a change in baseline firing following nerve transection contributed significantly to the increased responding of the motor cells to light stimulation, one would expect to find a significant correlation between baseline firing rate and the magnitude of the light-induced change in the number of action potentials. However, there was virtually no correlation between these 2 variables $(r=0.048$, n.s.). Thus, changes in the baseline excitability of the motor cells cannot account for the increase in light-induced excitation observed following nerve transections.

These results support 2 primary conclusions: First, brightlight input to pedal neck motor neurons from the eyes and rhinophores is functionally inhibitory; and second, blocking this inhibition unmasks an additional extraocular pathway from the periphery that mediates significant light-induced excitation to the same pedal motor neurons.

\section{Identification of an excitatory extraocular pathway}

Having determined that the optic and rhinophore nerves mediate functionally inhibitory input to pedal motor neurons, we wished to determine which of the remaining peripheral nerves carried the light-induced excitation observed in the previous experiments. To examine this question we assessed the effects of light on the same population of pedal motor neurons, using identical experimental procedures as before, with the exception that the optic and rhinophore nerves were transected $10 \mathrm{~min}$ prior to the beginning of the experiment. In this study, only motor neurons with a clear excitatory response to light were used. We found that peripheral nerves from the oral veil (nerves 


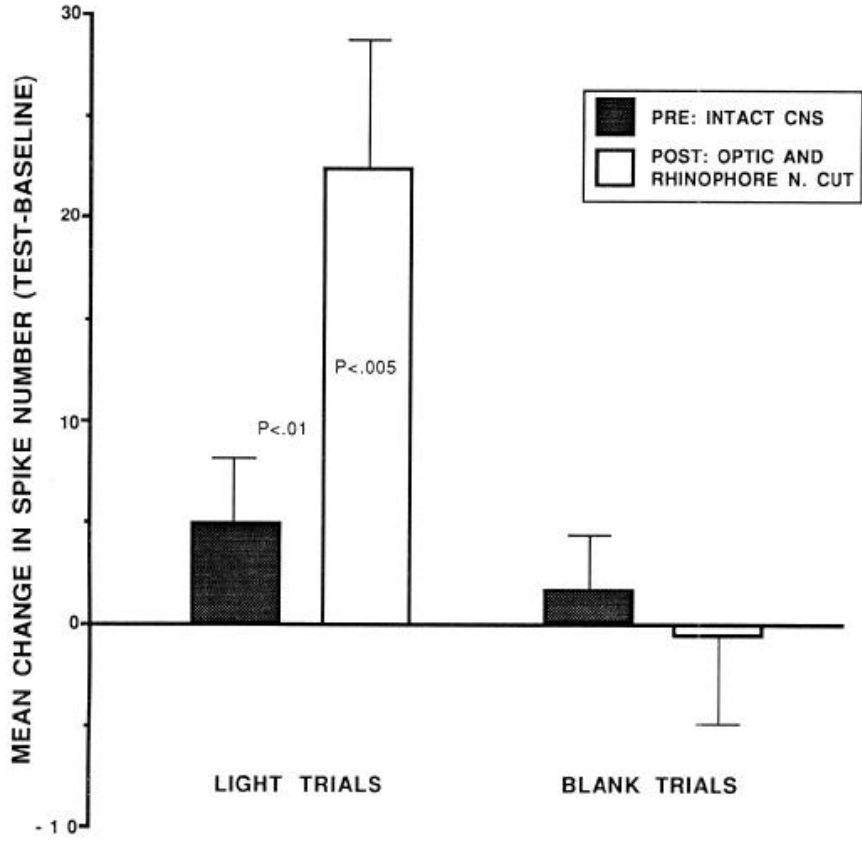

Figure 5. Summary of effects of optic and rhinophore nerve transection. Data are expressed as difference scores reflecting the mean change in spike number compared with the baseline period for light and blank trials; both before and after transection of the optic and rhinophore nerves $(n=10)$. Data from light trials show that, following nerve transection, motor cells exhibit a significant enhancement of their response to light $(p<0.005)$. Moreover, this posttransection light response is significantly greater than the response to light observed with the intact CNS $(p<0.01)$. Data from blank trials were not significant, showing that sampling bias cannot contribute to the results obtained on light trials.
C1-C3; Fig. 2) mediated a significant component of the lightinduced excitation. An example of our results is shown in Figure 6. Following transection of the optic and rhinophore nerves, light produced a large increase over baseline in the number of action potentials elicited in the motor neuron (an increase of $38 \%$; baseline action potentials $=87$; light trial $=120$; upper trace in Fig. 6). However, following bilateral transection of oral veil nerves $\mathrm{C} 1-\mathrm{C} 3$, light-induced excitation of the cell was abolished (baseline action potentials $=86$; light-trial $=78$; lower trace in Fig. 6). A summary of our results from 6 experiments (one pedal motor neuron per experiment) is shown in Figure 7. Data are expressed as the mean change in spike number (light trials - baseline). In the initial condition, only optic and rhinophore nerves were transected. This was followed by additional bilateral transections of nerves $\mathrm{C} 1-\mathrm{C} 3$. With the optic and rhinophore nerves cut, there was a clear light-induced increase of spikes above baseline $(\bar{x}=43.3 \pm 9.2)$. Following transection of nerves $\mathrm{C} 1-\mathrm{C} 3$, a between-condition analysis showed a significant decrease in the light-induced excitation of the pedal motor cells (from $\bar{x}=43.3$ spikes, \pm 9.2 ; to 1.2 spikes, \pm 8.7 ; $t[5]=4.98, p<0.005)$. In addition, a within-condition analysis showed that there was no longer a significant effect of light on the pedal motor neurons following transection of nerves $\mathrm{C} 1-\mathrm{C} 3$ $(t[5]=0.56$, n.s.). Finally, no significant changes in cell firing were observed during blank trials before and after cuts of $\mathrm{C1}-$ $\mathrm{C} 3(t[5]=0.93$, n.s. and $t[5]=0.56$, n.s., respectively), again ruling out the possibility that sampling bias produced these results.

As in the previous experiments that assessed the role of optic and rhinophore nerve input to pedal motor neurons, in the present experiments it was important to determine whether the loss of light-induced excitation following transection of nerves
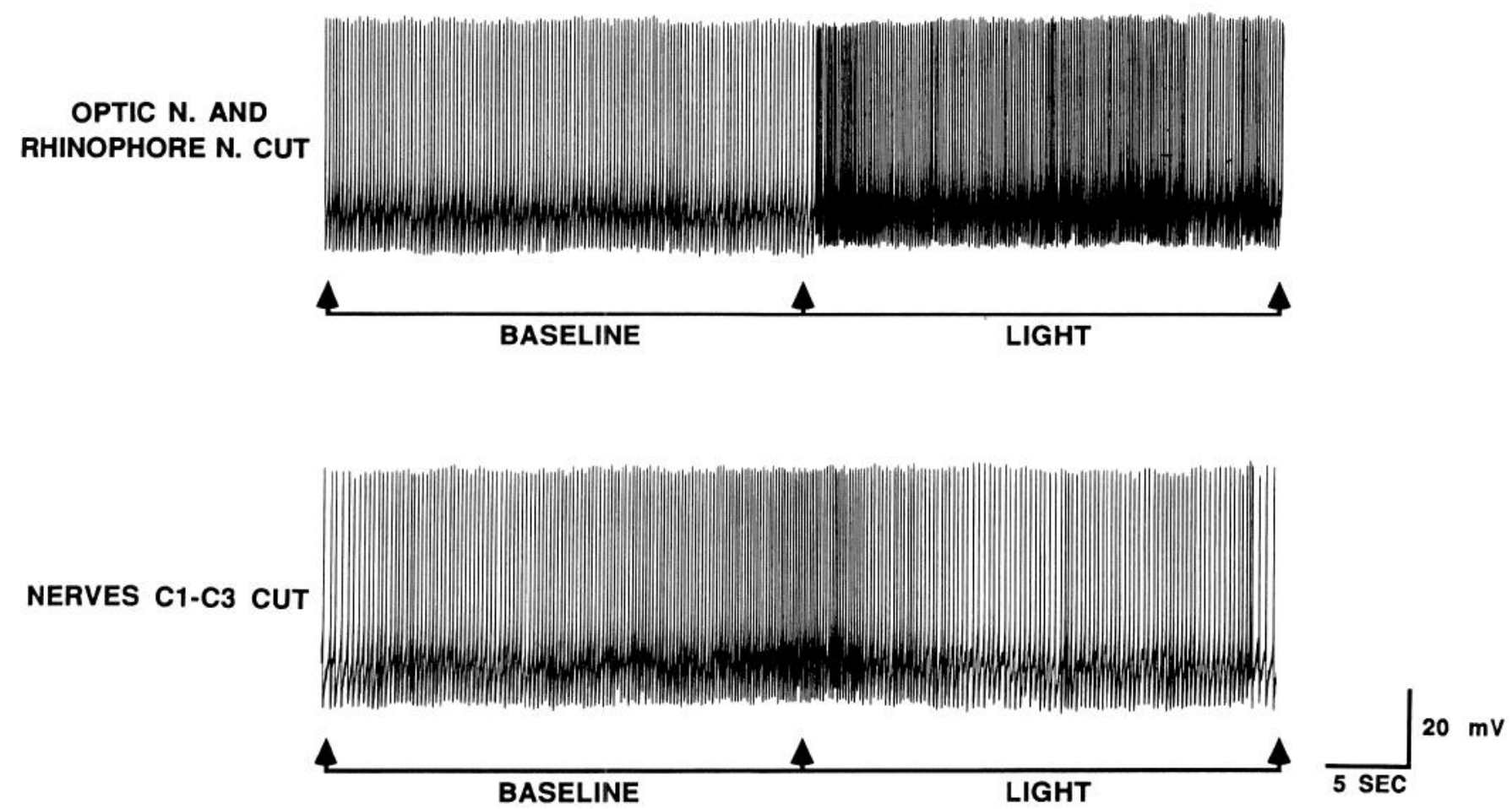

Figure 6. Role of oral veil nerves in light-induced excitation of pedal motor neurons. Traces are intracellular recordings from a pedal motor neuron. Top trace, With optic and rhinophore nerves cut, light produces an immediate and substantial increase in motor neuron firing rate. Bottom trace, Following transection of oral veil nerves $(\mathrm{Cl}-\mathrm{C} 3)$, the light-induced excitation observed above is abolished. 


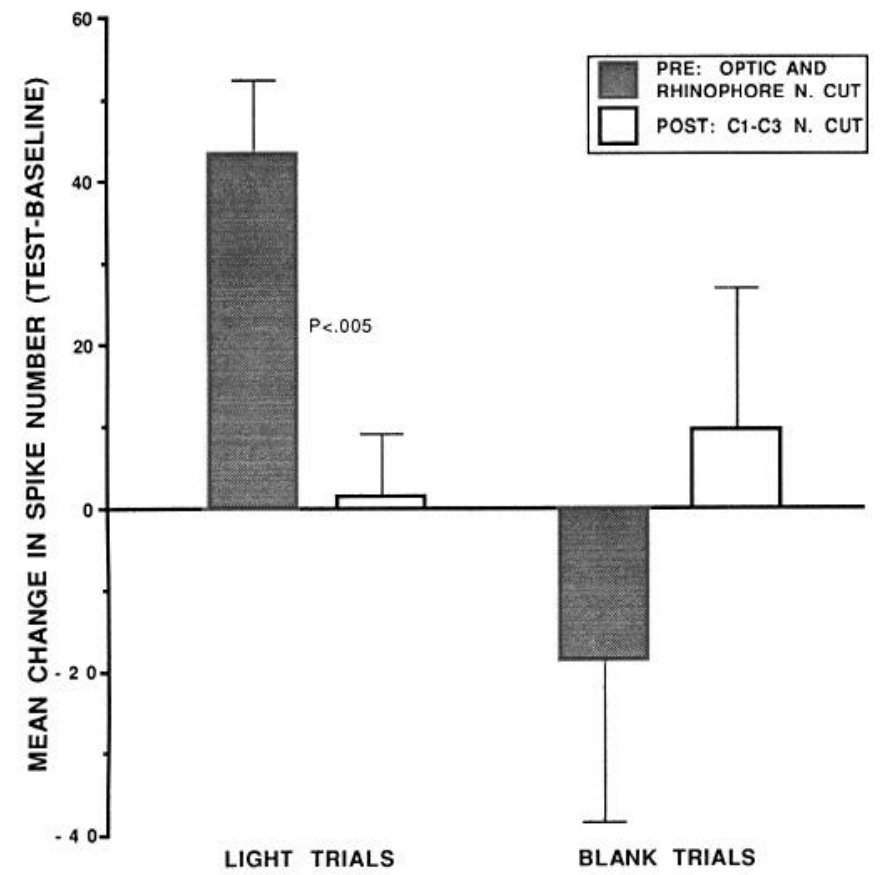

Figure 7. Summary of effects of oral veil nerve transection. Data are expressed as difference scores, as in Figure 5. Results from light trials $(n=6)$ show that following optic and rhinophore nerve transection, light produces marked excitation in pedal motor neurons (black bars) Following transection of the oral veil nerves $(\mathrm{C} 1-\mathrm{C} 3)$, there is a significant reduction in light-induced firing of the motor neurons $(p<0.005)$. Results from blank trials are not significant.

$\mathrm{C} 1-\mathrm{C} 3$ was due to an alteration in baseline firing following the transection. To examine this possibility we compared baseline firing rates (for both light and blank trials) before and after nerve cuts. There was no change in the mean baseline firing rates for the motor cells following transection of nerves $\mathrm{C} 1-\mathrm{C} 3$ (precut $\bar{x}$ baseline spikes $=59.9 \pm 23.9$; postcut $\bar{x}$ baseline spikes $=$ $59.6 \pm 19.5 ; t[5]=0.01$, n.s.). Moreover, there was no correlation between the change in light-induced excitation following transection of nerves $\mathrm{Cl}-\mathrm{C} 3$ and baseline firing rate of the cells ( $r=0.08$, n.s.).

In summary, our data show that there are at least 2 pathways conveying light information into the CNS that can modulate the excitability of neck motor cells in the pedal ganglion. One pathway, the optic and rhinophore nerves, has a strong, functionally inhibitory effect; the second pathway, nerves $\mathrm{Cl}-\mathrm{C} 3$, has a strong, functionally excitatory effect on the same pedal motor cells.

\section{Discussion}

Bright light as an aversive reinforcer in Aplysia

In our previous behavioral and EMG_studies of operant conditioning in Aplysia, bright light has been used as aversive reinforcement (Cook and Carew, 1986, 1989b). In the operant paradigm we employ, animals were suspended underwater by their parapodia in a testing chamber. During training, head movements to one side of the body were punished by exposing the animal to whole-field illumination. The lights remained on until the animal made a clear head-waving response to the unpunished (safe) side. Animals evidenced operant conditioning by spending significantly more time on the safe side during the test period compared with baseline (Cook and Carew, 1986). An important feature of this training procedure is that there is no directional information in the presentation of light: reinforcement was delivered uniformly in the test chamber, equally illuminating the whole body and the entire chamber. Thus, in order to acquire the operant task, the animals must learn that a particular behavior (e.g., head-waving to a particular side) results in the onset or offset of light; animals cannot acquire the task by determining the source of the light and moving along a light gradient to avoid it. This aspect of training is significant because the cellular analysis of the reinforcement pathway thus reduces to an examination of how the animals detect bright light, rather than how they determine the source of the light. This feature of the operant conditioning protocol greatly simplifies the task of characterizing the cellular properties of the reinforcement pathway.

Aplysia have both ocular and extraocular photoreceptors. The ocular receptors consist of a pair of bilaterally symmetric eyes located on the head near the base of the rhinophores; each eye contains approximately 4000 photoreceptor neurons (Jacklet, 1969a, 1973; Jacklet et al., 1972). Photic input from the eyes to the CNS is carried by the optic nerve which connects to the ipsilateral side of the cerebral ganglion (Jacklet and Geronimo, 1971; Jacklet 1976). In addition to supplying the CNS with photic input, the eyes also contain circadian pacemakers which innervate the entire CNS (Jacklet, 1969b; Eskin, 1971; Olson and Jacklet, 1985). Extraocular photoreceptors are found throughout the body. A set of particularly sensitive extraocular pathways project from the tips of the rhinophores, which respond to low intensities of light with roughly the same sensitivity as the eyes themselves (Chase, 1979; Jacklet, 1980). For this reason, in the present study we have combined the pathways from the eyes and rhinophores, considering them collectively as primary visual pathways. In our operant conditioning paradigm all sources of photic input to the CNS (ocular and extraocular) are exposed to the bright light stimulus.

\section{Light-induced excitation of pedal motor neurons}

Although many regions of the CNS are innervated by axons that carry information from photoreceptors (Jacklet, 1980; Olson and Jacklet, 1985), we were primarily interested in lightsensitive pathways that modulate the excitability of motor neurons involved in the operant response of head-waving. The cells we chose to examine were a small cluster of motor neurons (80$150 \mu \mathrm{m}$ in diameter), located in the dorsal pedal ganglion, near the statocysts. These cells mediate powerful contractions of the mesotegument of Aplysia (Hening et al., 1979). Moreover, these motor cells have recently been shown to be capable of expressing operant modification of their activity by bright-light reinforcement (Cook and Carew, 1988). Thus, these motor cells provided a sensitive vantage point from which to assess the effects of light in the operant response pathway. By identifying the source of light-induced modulation of these motor cells, we have been able to obtain clues as to which visual pathways are likely to be important for operant conditioning. However, we should emphasize that although these motor neurons clearly contribute to the head-waving response and are capable of operant modification (Cook and Carew, 1988), their specific role in operant conditioning remains to be established.

To determine the effects of light upon the pedal motor neurons, we compared the number of action potentials that occurred during a $30 \mathrm{sec}$ baseline (in the dark) with the number of action 
potentials which occurred during a $30 \mathrm{sec} \mathrm{light} \mathrm{trial} \mathrm{immediately}$ following baseline. We found that, with all peripheral input to the CNS intact, light had a modest excitatory effect upon the motor neurons. However, when the optic and rhinophore nerves were transected, a large increase in light-induced excitability was unmasked. These findings were surprising because a large portion of the photoreceptors in Aplysia are located in the eyes and thinophores. Moreover, optic input to the CNS is very extensive (Olson and Jacklet, 1985). Therefore, we had expected that bright light would affect the nervous system primarily through these pathways. Instead, these results show that with bright-light input from the eyes and rhinophores actually mediates functional inhibition of the pedal motor cells. One or more remaining extraocular pathways appeared to be responsible for mediating the excitatory effects of bright light. The extraocular pathways responsible for the dramatic increase in light-evoked activity were identified by subsequent transection of cerebral nerves $\mathrm{Cl}-\mathrm{C} 3$ which abolished the light-induced excitation. Another potential source of excitation could have come from direct effects of light on central neurons because it is known that many nerve cells in Aplysia can respond directly to light (Arvanitaki and Chalazonitis, 1961; Block and Smith, 1973). However, total isolation of central neurons from the periphery or transection of only nerves $\mathrm{Cl}-\mathrm{C} 3$ abolished the light-induced excitation, ruling out the possibility that direct illumination of the CNS mediates the increased responsiveness to light following transection of primary optic pathways (optic and rhinophore nerves).

The effects of nerve transection on light-induced excitation of pedal motor neurons raise important questions about the functional organization of the visual system of Aplysia. For example, why is the CNS of Aplysia organized such that visual input from the most anterior portion of the head (the oral veil) excites pedal motor neurons, while photoreceptors located somewhat more posteriorly, in the eyes and rhinophores, mediate inhibition of the same cells? It is possible that this organization of extraocular photic input may convey directional information about light. Since in our experiments the light stimulus is whole-field illumination, such directional consequences of light for motor output would not be detected. Moreover, it is known that an extraocular pathway can mediate photic entrainment of the locomotor rhythm in Aplysia (Lickey et al., 1977). Thus, the extraocular pathways in Aplysia provide an important source of sensory information that is capable of modulating a variety of behavioral response systems.

A critical question raised by our findings concerns the role played by the 2 visual pathways we have identified, the eye and rhinophore nerves and the oral veil nerves, in mediating reinforcement during operant conditioning of head-waving. The fact that the optic and rhinophore nerves mediate an inhibitory effect to head-waving motor neurons in response to bright light raises the interesting (and counterintuitive) prediction that these pathways may not be required for operant conditioning to occur. Consistent with this hypothesis, we have found that chronic transection of the optic and rhinophore nerves does not interfere with the acquisition of operant conditioning. In fact, the results suggest that the learning is actually improved compared with sham-operated animals (Cook and Carew, 1987; D. G. Cook and T. J. Carew, unpublished observations). These results further suggest that the oral veil nerves $(\mathrm{C} 1-\mathrm{C} 3)$, which mediate an excitatory effect in pedal motor neurons, may play a significant role in the learning. Behavioral experiments have recently confirmed this prediction: Chronic bilateral transections of nerves C1-C3 abolish the ability of animals to acquire the operant conditioning (Cook and Carew, 1989c; Cook and Carew, unpublished observations).

In conclusion, having identified 2 functionally distinct visual pathways that modulate (in opposite directions) the activity of motor neurons in the operant response pathway, it will now be necessary to characterize sensory ncurons and interncurons underlying these pathways and to determine the degree to which they contribute to the mediation of reinforcement in our learning task. We have begun this analysis by combining double-label backfills of cerebral and pedal nerves with physiological and anatomical characterization of individual pedal motor neurons that contribute to the generation of the operant response (Kuenzi and Carew, 1988; D. G. Cook, A. C. Fish, and T. J. Carew, unpublished observations). The ultimate goal of this combined effort is to identify sites of synaptic convergence between reinforcement pathways on the one hand and operant response pathways on the other. Such convergent sites will provide candidate loci for the synaptic modifications that underlie operant conditioning of head-waving in Aplysia.

\section{References}

Arvanitaki, A., and N. Chalazonitis (1961) Excitatory and inhibitory processes initiated by light and infra-red radiations in single identifiable nerve cells (giant ganglion cells of Aplysia). In Nervous Inhibition: International Symposium on Nervous Inhibition, E. Florey, ed., pp. 194-231, Pergamon, New York.

Block, G., and J. T. Smith (1973) Cerebral photoreceptors in Aplysia. Comp. Biochem. Physiol. 46A: 115-121.

Carew, T. J., E. T. Walters, and E. R. Kandel (1981) Associative learning in Aplysia: Cellular correlates supporting a conditioned fear hypothesis. Science 211: 501-504.

Chase, R. (1979) Photic sensitivity of the rhinophore in Aplysia. Can. J. Zool. 57: 698-701.

Cook, D. G., and T. J. Carew (1986) Operant conditioning of headwaving in Aplysia. Proc. Natl. Acad. Sci. USA 83: 1120-1124.

Cook, D. G., and T. J. Carew (1987) Cellular analysis of the reinforcement pathway in operant conditioning of head-waving in $\mathrm{Aply}$ sia. Soc. Neurosci. Abstr. 13: 815 .

Cook, D. G., and T. J. Carew (1988) Operant conditioning of identified neck muscles and individual motor neurons in Aplysia. Soc. Neurosci. Abstr. 14: 607.

Cook, D. G., and T. J. Carew (1989a) Operant conditioning of headwaving in Aplysia. I. Identified muscles involved in the operant response. J. Neurosci. 9: 3097-3106.

Cook, D. G., and T. J. Carew (1989b) Operant conditioning of headwaving in Aplysia. II. Contingent modification of electromyographic activity in identified muscles. J. Neurosci. 9: 3107-3114

Cook, D. G., and T. J. Carew (1989c) Identification of reinforcement pathways necessary for operant conditioning in Aplysia. Soc. Neurosci. Abstr. 14: (in press).

Eskin, A. (1971) Properties of the Aplysia visual system: In vitro entrainment of the circadian rhythm and centrigual regulation of the eye. Z. vgl. Physiol. 74: 353-371.

Hening, W. A., E. T. Walters, T. J. Carew, and E. R. Kandel (1979) Motorneuronal control of locomotion in Aplysia. Brain Res. 179:231253.

Jacklet, J. W. (1969a) Electrophysiological organization of the eye of Aplysia. J. Gen. Physiol. 53: 21-42.

Jacklet, J. W. (1969b) Circadian rhythm of optic nerve impulses recorded in darkness from isolated eye of Aplysia. Science 164: 562563.

Jacklet, J. W. (1973) Model for the circadian neuronal activity of the eye of Aplysia. Physiologist 16: 352.

Jacklet. J. W. (1976) Dye marking neurons in the eye of Aplysia. Comp. Biochem. Physiol. 55: 373-377.

Jacklet, J. W. (1980) Light sensitivity of the rhinophores and eyes of Aplysia. J. Comp. Physiol. 136: 257-262. 
Jacklet, J. W., and J. Geronimo (1971) Circadian rhythm: Population of interacting neurons. Science 174: 299-302.

Jacklet, J. W., R. Alvarez, and B. Bernstein (1972) Ultrastructure of the eye of Aplysia. J. Ultrastruct. Res. 38: 246-261.

Kuenzi F. M., and T. J. Carew (1988) A biomechanical analysis of the head-waving response in Aplysia. Soc. Neurosci. Abstr. 14: 1000.

Lickey, M. E., J. A. Wozniak, G. D. Block, D. J. Hudson, and G. K. Augter (1977) The consequences of eye removal for the circadian rhythm of behavioral activity in Aplysia. J. Comp. Physiol. 118: 121143.
Newby, N. A. (1972) Habituation to Light and Spontaneous Activity in the Isolated Siphon of Aplysia: The Effects of Synaptically Active Pharmacological Agents. Ph.D. dissertation, Case Western Reserve University, Cleveland, Ohio.

Olson, L. M., and J. W. Jacklet (1985) The circadian pacemaker in the Aplysia eye sends axons throughout the central nervous system. J. Neurosci. 5: 3214-3227. 